\title{
Anterior cruciate ligament integrity in osteoarthritis of the knee in patients undergoing total knee replacement
}

\author{
M. J. M. Douglas · J. D. Hutchison • \\ A. G. Sutherland
}

Received: 15 October 2009/ Accepted: 9 August 2010/Published online: 11 September 2010

(C) The Author(s) 2010. This article is published with open access at Springerlink.com

\begin{abstract}
Background Anterior cruciate ligament (ACL) rupture has been implicated in the development of knee osteoarthritis (OA). This study aimed at determining the incidence of prior ACL deficiency in patients undergoing total knee replacement (TKR), the effect of prior ACL deficiency on function and the macroscopic and microscopic appearance of the ligament.

Materials and methods A total of 95 patients undergoing elective TKR for OA were recruited. Pre-operative knee assessment included questionnaires and KT1000 testing. The ACL was examined macroscopically at TKR in all patients, and $10 \mathrm{ACL}$ specimens were examined histologically.

Results The ACL was absent in $12 \%$ of the patients. There was no significant correlation between the preoperative assessment or function and operative findings. The ACL samples all demonstrated degenerative change of varying severities.

Conclusion ACL deficiency is uncommon in patients undergoing TKR for OA, and does not worsen pre-operative function.
\end{abstract}

Keywords Osteoarthritis - Anterior cruciate ligament · Arthroplasty · Degeneration · Trauma

M. J. M. Douglas · J. D. Hutchison · A. G. Sutherland ( $\square)$ Department of Orthopaedics, University of Aberdeen, Polwarth Building, Foresterhill, Aberdeen AB25 2ZD, Scotland, UK

e-mail: ort025@abdn.ac.uk

\section{Introduction}

Anterior cruciate ligament (ACL) rupture is a significant injury, and ACL deficiency is known to lead to progressive degenerative changes in the knee, resulting in the development of premature secondary osteoarthritis (OA). Deficiency of the ACL changes the kinematics at the knee joint and results in abnormal loading and increased translation of the tibio-femoral joint, which in turn results in articular cartilage damage and development of osteoarthritis [1-3], particularly in the lateral compartment [4].

The relationship between ACL injury and OA remains to be fully clarified. It has been reported that whilst $19 \%$ [5] to $23 \%$ [6] of patients with symptomatic OA may have a deficient ACL on MRI scanning, the majority have no previous history of trauma to cause such damage. A direct observational study found ACL deficiency in 39\% of 107 patients undergoing total knee replacement (TKR) [4]. It was suggested that osteophyte formation, with narrowing of the intercondylar notch, may lead to impingement on and attrition of the ACL, which also undergoes age-related degeneration [7].

Anterior cruciate ligament deficiency as a result of either trauma or attrition was not a common finding at TKR in a short pilot study that we undertook (one out of ten patients observed sequentially, unpublished data). We hypothesised that ACL deficiency would be an uncommon finding in a larger, prospective observational study in patients with end-stage osteoarthritis, and that ACL deficiency would not significantly alter their functional levels. An observational study was undertaken in patients undergoing TKR for end-stage OA, to assess clinical findings with function (general, knee OA-specific and ACL-specific), and to relate that to the macroscopic appearance of the ACL at surgery. 


\section{Materials and methods}

Patients between the ages of 50 and 85 years who were admitted to our unit for elective total knee replacement for primary or secondary osteoarthritis and were able to give consent were eligible for inclusion. Those with a past history of rheumatoid arthritis were excluded. Ethical approval was received before commencement of the study from the North of Scotland Local Research Ethics Committee and informed consent was received from every patient prior to participation in the study, which was performed in accordance with the ethical standards of the 1964 Declaration of Helsinki, as revised in 2000.

One hundred and seventeen consecutive patients were invited to take part in the study between January and May 2008. Five patients had their planned operation changed from a TKR, three patients had their operations cancelled due to medical problems and twelve patients declined to take part in the study. This left 95 patients who participated in the study. Demographic details were collected for all patients, including any history of previous significant injury to the patient's operative knee and symptoms specific to OA and ACL deficiency.

In ten sequential patients at the beginning of the study, additional permission was sought to harvest the ACL (normally discarded at the time of surgery) to allow histological examination of the ACL for evidence of degenerative changes. These patients were asked to join this ACL histology sub-group sequentially, and no attempt was made to recruit patients according to their expected ACL status and all agreed to participate in this part of the study.

Assessment of pre-operative knee function

The pre-operative knee function of each patient was assessed subjectively using a self-administered patient questionnaire, consisting of the Mohtadi ACLQuality of Life (ACLQoL) questionnaire, Oxford Knee Score (OKS) and Short Form-12 (SF-12) Health survey.

$A C L Q o L$

This is a measure specifically designed to evaluate the quality of life of patients suffering from chronic ACL deficiency [8]. It consists of questions that are each answered by a $100 \mathrm{~mm}$ visual analogue scale, producing individual scores that are used to produce an overall average score from 0 (poor) to 100 (best function). This is the only disease-specific measure for function related to ACL deficiency, although it was not primarily developed for use in an elderly, arthritic population.

\section{$O K S$}

This is a validated measure of pain and function in patients with knee arthritis $[9,10]$. It consists of 12 questions, each scored from 1 to 5, with a score of 1 indicating least difficulty or severity to a score of 5 indicating the most difficulty or severity. The total overall score can be between 12 (indicating least difficulty) to a maximum score of 60 (indicating severe difficulty in knee function).

\section{Short form-12 health survey (SF-12)}

This is a short measure of general health [11]. The questions are answered via multiple choice options and are grouped to produce eight health scales in two main categories, physical health and mental health. The questions are then each scored to produce the physical and mental component summary scores.

\section{International knee documentation committee assessment form (IKDC)}

The patients' pre-operative knee was assessed in terms of effusion, passive motion deficit and ligament examination utilising the IKDC form. This is a categorical assessment tool that can be used pre- and post-operatively and also at follow-up and was designed to be a standardised form for the assessment of the results of treatment of knee ligament injuries [12, 13]. It provides the best-available way of undertaking a structured clinical examination of the knee. For the purposes of this study, the form was used preoperatively to evaluate the patients' knees for evidence of ligament laxity and range of motion, each section being graded either 'normal', 'nearly normal', 'abnormal' or 'severely abnormal', and included standardised clinical examination and laxity assessment with the KT-1000 arthrometer.

\section{Assessment of macroscopic status of the ACL}

At the time of knee replacement, the macroscopic status of the ACL was recorded as either normal (where the ACL had normal appearance macroscopically, with no fibre disruption apparent), abnormal but present (where there were surviving ACL fibres without a normal appearance) or absent, on a standardised tick-box form by the operating surgeon who examined the ACL at the time of surgery.

Ten ACL specimens were taken at surgery from the ACL histology sub-group patients. These were examined histologically for evidence of degenerative change following a similar method as detailed in a previous paper by Insall [7]. The presence of specific degenerative changes 
was noted, in particular any evidence of loose fibrous connective tissue, cystic appearance of the tissue, occurrence of granular substance, myxoid appearance of the tissue, chondroid metaplasia, calcium pyrophosphate (CPPD) crystals and mucinous degeneration. Short descriptive reports were provided for the findings of each.

\section{Statistical analysis}

Advice on the statistical analysis was taken, prior to commencement, from the Department of Public Health, University of Aberdeen. As this was an observational study, no power calculation was carried out. Statistical analyses were carried out using the Statistical Package for the Social Sciences (SPSS) version 5.0 (SPSS, Inc, Chicago, IL, USA). Total and mean scores for the ACLQoL, OKS were calculated. The results from these questionnaires were normally distributed, enabling the means (and 95\% confidence intervals (CI)) to be compared using a oneway ANOVA. The SF-12 results were not normally distributed so are presented as median and interquartile range (IQR), and this required the use of a non-parametric test, the Kruskal-Wallis test to compare the results from the ACLQoL and SF-12.

Statistical analysis of the categorical data was carried out using Pearson chi square tests or Pearson correlation. In cases where the results contained a low count or the variables being compared contained unequal numbers of categories, a Fisher's exact test was used to examine the correlations. In all cases the value for the test and the $P$ value were reported. Significance was set as $P<0.05$.

\section{Results}

Ninety-five patients completed recruitment and are included in analysis. Their mean age was 68 years (53-82), and $56 \%$ were men. The mean age of the ACL histology subgroup was 70 years (range $55-79$ years) and $60 \%$ of the group were men.

Of the 95 patients recruited, $62(65 \%)$ had no history of a previous significant injury, and none had undergone a previous open meniscectomy. Thirty-three patients (35\%) had a history of previous injury, but none were aware of a specific diagnosis of ACL injury, and none had such a diagnosis recorded in their case notes. No further attempt was made to classify the possible causes of the end-stage oateoarthritis present in these patients. With regard to knee symptoms possibly attributable to ACL deficiency, $65 \%$ (62) of the patients reported instability in their operative knee, $31 \%$ (29) reported locking and hyperextension was only reported by $3.2 \%$ [3] of the patients, with most having a fixed flexion deformity in their operative knee as a result of OA.

\section{IKDC assessment}

All $95(100 \%)$ patients were examined clinically by the researcher (MJMD) using the IKDC knee evaluation form. The descriptive data for each problem area of the knee evaluated by the form is shown in Table 1. Examination of the ACL by Lachman and Anterior Drawer tests and KT-1000 arthrometer measurement was graded as normal or nearly normal in nearly all patients.

In this study, the majority of patients (77\%) had an abnormal opposite (non-operative) side, making a side-toside difference for KT-100-measured laxity difficult to interpret. The pure anterior translation of the study knee was therefore assessed in isolation, taking the IKDC classifications of translation to give a "worst case scenario" analysis in comparison with a nominally normal translation on the contralateral side of $2 \mathrm{~mm}$, in which case 68 $(71.6 \%)$ of the index knees were normal or nearly normal. Table 2 summarises the anterior translation grading findings, cross-referenced with the surgical findings. There were no statistically significant association between anterior translation as graded in this way with extension loss (Pearson chi square test $=13.76, P=0.13$ ) or flexion loss (Pearson chi square test $=12.84, P=0.17$ ).

\section{Patient questionnaires}

The mean ACLQoL scores, $($ mean $=37.11,95 \%$ CI 33.41-40.82) suggested that most of the patients in the study had a poor ACL related quality of life with regard to their index knee. The mean OKS was 39.95 (95\% CI 38.34-41.60). The majority of patients, 47 (49.5\%), were graded as severe. The median SF-12 Mental component score (SF-12MCS) was 53.00 (IQR 18.80), and the median
Table 1 IKDC clinical examination grading of patient group $(n=95)$

\begin{tabular}{lllcl}
\hline & Normal & Nearly normal & Abnormal & Severely abnormal \\
\hline Effusion (\%) & $30(31.6 \%)$ & $39(41.1 \%)$ & $25(26.3 \%)$ & $1(1.1 \%)$ \\
Manual Lachman $(\%)$ & $47(50.0 \%)$ & $43(45.7 \%)$ & $4(4.3 \%)$ & 0 \\
Anterior drawer $(\%)$ & $71(74.7 \%)$ & $20(20 \%)$ & $5.3(5.3 \%)$ & 0 \\
KT-1000 $(\%)$ & $62(65.3 \%)$ & $27(28.4 \%)$ & $6(6.3 \%)$ & 0 \\
\hline
\end{tabular}


Table 2 ACL gross appearance cross-referenced with anterior translation as measured by KT-1000 arthrometer, relative to a nominal contralateral normal of $2 \mathrm{~mm}$

\begin{tabular}{lllllll}
\hline & & \multicolumn{2}{l}{ KT-1000 Anterior translation grade } & \multicolumn{2}{c}{ Total } \\
\cline { 3 - 6 } & & Normal & $\begin{array}{l}\text { Nearly } \\
\text { normal }\end{array}$ & Abnormal & $\begin{array}{c}\text { Severely } \\
\text { abnormal }\end{array}$ \\
\hline $\begin{array}{l}\text { ACL gross } \\
\text { appearance }\end{array}$ & $\begin{array}{c}\text { Normal } \\
\text { Abnormal but } \\
\text { present }\end{array}$ & 11 & 11 & 7 & 1 & $51(54.8 \%)$ \\
& Absent & 2 & 7 & 3 & 0 & $30(32.3 \%)$ \\
Total & $30(31.6 \%)$ & $38(40.0 \%)$ & $23(24.2 \%)$ & $2(2.1 \%)$ & $93(97.9 \%)$ \\
\hline
\end{tabular}

SF-12 physical component score (SF-12PCS) was 29.57 (IQR 11.86). The distribution of the scores indicated that the majority of the patients suffered significant physical and mental dysfunction associated with OA of their knee.

Operative findings and histology

Operative data was available for $93(97.9 \%)$ of the patients, and is summarised in Table 2. Only 12 patients had an absent ACL, five of whom gave a history suggestive of previous ACL rupture. Histologically, the ACL specimens showed some evidence of degenerative change in all ten specimens. The severity of degenerative change was categorised as mild (in 6 of 10), moderate or marked (seen in 4 of 10). Myxoid degeneration was observed in seven of the specimens. Chondroid metaplasia was evident in four specimens and cyst formation was also evident in four specimens. Other appearances of the ACL specimens that were noted less frequently in the pathology reports included the presence of fibrosis which was noted in two specimens and raised the possibility of previous ligament injury in these specimens. Synovial hyperplasia was noted in four specimens. In one such specimen it was present along with synovial hypertrophy, a lymphoplasmacytic inflammatory cell infiltrate, small lymphoid aggregates and haemosiderin deposition which suggested the appearance of resolving chronic synovitis. In another specimen showing synovial hyperplasia, occasional miniscule spicules of bony detritus were present.

There was no statistically significant association between history of a previous index injury and the status of the ACL at the time of TKR. (Pearson chi square test $=0.260, \quad P=0.878)$. Clinical examination and KT-1000 assessment did not predict the gross appearance of the ACL (ACL Surgical Grade and manual Lachman grade (Fisher's exact test $=5.70, P=0.18$ ), Anterior Drawer test (Fisher's exact test $=2.58, \quad P=0.62$ ), KT-1000 side-to-side difference (Pearson Chi square = $3.55, P=0.74)$, or pure $\mathrm{KT}-1000$ anterior translation (Fisher's exact test 4.14, $P=0.72$ ). Patients with an absent ACL had a higher (i.e., better) ACL QoL (one way ANOVA

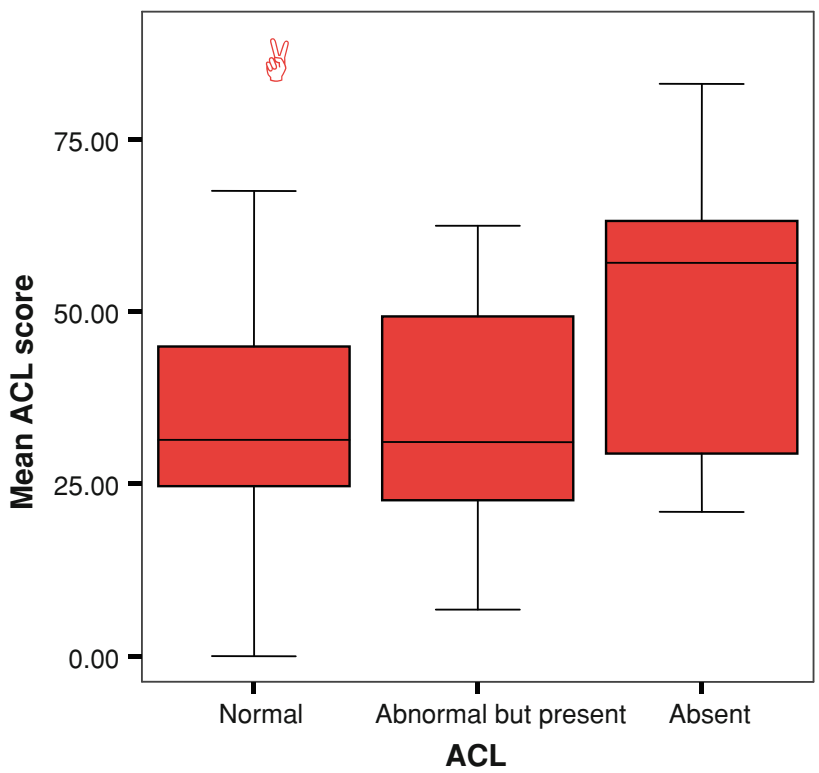

Fig. 1 Mean ACLQoL score versus ACL operative status for main group

$(P=0.03$, see Fig. 1$)$. There was no statistically significant relationship between OKS score or category and ACL status (One way ANOVA, $P=0.164$; Pearson chi square $=0.663, P=0.718)$.

There was no significant correlation between the SF-12 physical component scores for the ACL main group and the surgical status of the ACL (Kruskal-Wallis test $=1.70$, $P=0.43$ ). There was a significant correlation between the SF-12 mental component score for the ACL main group and the surgical status of the ACL (Kruskal-Wallis test $=7.59 P=0.022$ ) with those with an abnormal or absent ACL having worse SF12 mental component scores. The ACLQoL score correlated with the SF12 physical component scores (Pearson correlation $=0.308$, $P=0.009)$ but not with the SF12 mental component scores (Pearson correlation $=0.229, P=0.057$ ).

ACL surgical status correlated poorly with histological status (Pearson Chi square test $=0.625, P=0.429$ ). 


\section{Discussion}

This is the first study, to the best of our knowledge, which has attempted to assess the integrity of the ACL in patients undergoing TKR by means of detailed questionnaire and examination (including KT-1000 assessment), and then correlating these findings with the intra-operative findings at the time of TKR. Several aspects of the relationship between ACL deficiency and osteoarthritis of the knee have been investigated by others using various methods. However, these have produced a variety of results, which have frequently conflicted with each other $[4,6,7]$. It has been suggested that ACL deficiency leads to articular cartilage damage and osteoarthritic changes in the knee [4]. Other studies $[5,6]$ have shown that up to $39 \%$ of patients may have a deficient ACL on MRI scanning, often without specific prior injury trauma. It has been suggested that that intercondylar notch impingement may then lead to attrition and rupture of the ACL. It is important to note that this proposed association has never been confirmed.

In our study, only $13 \%(12 / 95)$ of the patients undergoing TKR had an absent ACL, which is less than the 23\% suggested by Hill et al. [6] in their MRI-based study. Only five of our patients with an absent ACL at the time of surgery had a history indicating that they had sustained a previous index injury. The ACL is certainly affected in degeneration of the knee, as borne out by our histological results, but it does not appear that this degeneration causes a significant risk of non-traumatic rupture except in a small minority of cases (7\%). A majority of patients $(65 \%)$ reported experiencing instability and $31 \%$ reported experiencing locking in their knee, but these findings cannot be directly attributed to ACL deficiency with any certainty as both can be experienced as a consequence of the OA disease process.

Clinical assessment of the knee by Lachman's and anterior drawer tests and KT-1000 anterior translation measurements did not predict the appearance of the ACL at the time of surgery. The side-to-side difference in KT-1000 arthrometer measurements (instrumented Lachman's) was found to be difficult to interpret in patients with two abnormal knees ( $77 \%$ in the study) and is not recommended for use in future studies. Instead, the index knee was compared to a nominal normal of $2 \mathrm{~mm}$ of translation on the contralateral side, producing a worst-case-scenario side-to-side difference. This measurement categorised $24.2 \%$ of the index knees as abnormal and, in those patients with an absent ACL at the time of surgery, 25\% (3/12)were graded as "abnormal" by this assessment (Table 2). In many patients, the KT-1000 predicted ACL deficiency that was not found (of 25 abnormal or severely abnormal laxity measurements, 14 ACLs had normal appearance), or failed to predict ACL deficiency when it was present (of 42 patients with abnormal or absent ACL, 31 had normal or nearly normal laxity measurements). While it might be expected that joint stiffness, and development of osteophytes, might lead to less knee laxity in the presence of ACL deficiency, this is not always the case and in many cases, knees with marked laxity had macroscopically normal ACLs.

ACL status did not correlate with pre-operative knee function, as assessed by the patient questionnaires. The ACLQoL questionnaire was found to have difficulties when assessing the pre-operative knee function in a study population which is markedly younger than the age range of the patients in the original description of the tool [8]. Therefore, it is not recommended for use in further studies examining the link between ACL status and knee OA in elderly patient groups. The OKS was useful in assessing knee function but is not specific to ACL deficiency and therefore the finding that the majority (49.5\%) of the patients were graded as having a severe loss of knee function could not be attributed to ACL deficiency, and is more in keeping with advanced OA. The SF-12 Health Survey, although found to provide useful information, was also found to be problematic with patients reporting that it was repetitive and finding some of the questions intrusive.

The strengths of our study are that this is a sequential observational study of patients with end-stage OA, with a structured pre-operative assessment by questionnaire and examination, combined with a direct assessment of the macroscopic appearance of the ACL at TKR. As it is observational rather than longitudinal, it is not possible to be absolutely certain about the mechanism of ACL failure where it has occurred. The use of the ACL QoL measure was problematic, and we would not advocate its use in an elderly population. There is no defined grading system for the appearance of the ACL, so our system of Normal, present but abnormal and absent was a pragmatic but not previously validated attempt to so grade the ACL.

In summary, our results do not support the suggestion that ACL rupture in OA of the knee is common, or that degenerative change, rather than traumatic injury, is responsible for ACL insufficiency that can lead to OA development. It is difficult to predict the gross appearance of the ACL from pre-operative clinical examination, including KT-1000 evaluation.

Acknowledgments With thanks to Professor SD Heys, University of Aberdeen, for advice on redrafting of manuscript.

\section{Conflict of interest None.}

Open Access This article is distributed under the terms of the Creative Commons Attribution Noncommercial License which permits any noncommercial use, distribution, and reproduction in any medium, provided the original author(s) and source are credited. 


\section{References}

1. Louboutin H, Debarge R, Richou J, Selmi TA, Donell ST, Neyret $\mathrm{P}$ et al (2009) Osteoarthritis in patients with anterior cruciate ligament rupture: a review of risk factors. Knee 16:239-244

2. Beynnon BD, Fleming BC, Labovitch R, Parsons B (2002) Chronic anterior cruciate ligament deficiency is associated with increased anterior translation of the tibia during the transition from non-weightbearing to weightbearing. J Orthop Res 20:332-337

3. Frank CB, Jackson DW (1997) The science of reconstruction of the anterior cruciate ligament. J Bone Joint Surg Am 79: $1556-1576$

4. Lee GC, Cushner FD, Vigoritta V, Scuderi GR, Insall JN, Scott WN (2005) Evaluation of the anterior cruciate ligament integrity and degenerative arthritic patterns in patients undergoing total knee arthroplasty. J Arthroplasty 20:59-65

5. Amin S, Guermazi A, Lavalley MP, Niu J, Clancy M, Hunter DJ et al (2008) Complete anterior cruciate ligament tear and the risk for cartilage loss and progression of symptoms in men and women with knee osteoarthritis. Osteoarthritis Cartilage 16: 897-902

6. Hill CL, Seo GS, Gale D, Totterman S, Gale ME, Felson DT (2005) Cruciate ligament integrity in osteoarthritis of the knee. Arthritis Rheum 52:794-799
7. Cushner FD, La Rosa DF, Vigorita VJ, Scuderi GR, Scott WN, Insall JN (2003) A quantitative histologic comparison: ACL degeneration in the osteoarthritic knee. J Arthroplasty 18:687-692

8. Mohtadi N (1998) Development and validation of the quality of life outcome measure (questionnaire) for chronic anterior cruciate ligament deficiency. Am J Sports Med 26:350-359

9. Dawson J, Fitzpatrick R, Murray D, Carr A (1998) Questionnaire on the perceptions of patients about total knee replacement. J Bone Joint Surg Br 80:63-69

10. Whitehouse SL, Blom AW, Taylor AH, Pattison GT, Bannister GC (2005) The Oxford knee score; problems and pitfalls. Knee 12:287-291

11. Gandek B, Ware JE, Aaronson NK, Apolone G, Bjorner JB, Brazier JE et al (1998) Cross-validation of item selection and scoring for the SF-12 health survey in nine countries: results from the IQOLA project. International Quality of Life Assessment. J Clin Epidemiol 51:1171-1178

12. Hefti F, Muller W, Jakob RP, Staubli HU (1993) Evaluation of knee ligament injuries with the IKDC form. Knee Surg Sports Traumatol Arthrosc 1:226-234

13. Brinker MR, Garcia R, Barrack RL, Timon S, Guinn S, Fong B (1999) An analysis of sports knee evaluation instruments. Am J Knee Surg 12:15-24 